Journal of the Operations Research

Society of Japan

Vol. 44, No. 4, December 2001

\title{
A DYNAMIC MODEL FOR A TWO-CABIN YIELD MANAGEMENT WITH FREE UPGRADING DECISION
}

\author{
Peng-Sheng You \\ National Taiwan University of Science and Technology
}

(Received April 24, 2000; Final June 4, 2001)

\begin{abstract}
It is a current airline industry practice to upgrade passengers from the economy cabin to the business cabin at no additional cost to the passengers. Incorporating this practice, this paper deals with a single-flight-leg multi-fare class seat inventory control problem. A discrete-time dynamic programming model for finding the optimal booking policy is developed. It is found that the booking policy can be reduced to some set of critical values.
\end{abstract}

\section{Introduction}

The airline business is an extremely competitive market. Within this competitive market, it has become important to develop strategies to improve revenues. One of the major strategies is the seat inventory control.

Since the marginal cost of carrying an additional economy/business passenger is relatively low when compared to the high fixed cost incurred on a flight, the improved load factor resulting from additional passengers can produce a significant increase in the total revenue. Thus, instead of departing with many vacant seats, airlines will try to sell all of the seats. A common strategy to increase sales is to classify a pool of identical seats into several fare classes through the application of restrictions or service on tickets [2]. Under this strategy, an identical seats in a certain cabin are sold at a variety of prices. Airlines can never be certain what types of booking requests will appear in the future. If most of the customers' booking requests are accepted regardless of the fare class, an airline may lose a lot of customers who are willing to pay higher fares. On the other hand, if airlines reject most of the lower fare booking requests, they run the risk of taking off with many vacant seats. Hence, a problem associated with management arises in the seat inventory control (i.e., what are the suitable booking limits with respect to different booking status).

Airline seat inventory control is an aspect of yield management. Several wonderful introductions to the airline yield management problem exist in the literature $[2,8,9,14]$. In addition, a number of models (e.g. [1], [3], [4-7], [10-12], [15-17]) have been proposed to determine the booking limit for different types of seat inventory control problems.

Using a method called the marginal seat revenue approach, Littlewood [11] applied a two-fare class model. Belobaba [3] furthered this work and proposed a general model with multiple fare classes, assuming that the booking process is sequentially monotonic, that is the lower value fare was assumed to be booked before the higher value fare. By the same assumption, Curry [6] developed a multiple fare class model using the mathematical programming approach. Wollmer [15] dealt with the multiple fare class model and introduced an algorithm for computing the optimal booking policy. Brumelle, McGill, Oum, Sawaki, 
and Tretheway [4] dealt with a multiple fare class problem by formulating a revenue function for both discrete and continuous probability distributions of demand, and the conditions revealed a concave revenue function. In addition to the previous research, Robinson [12] dealt with a model with multiple fare classes, assuming the booking process was sequentially nonmonotonic.

In another approach, Gerchak, Parlar, and Yee [7] used the dynamic approach to deal with a two-fare class model in which demands are modeled as a discrete time stochastic process. The assumption that demands are stochastic eliminates the need for the additional assumption that the demand from the different fare classes arrives sequentially. An important outcome of the work [7] is that the booking policy parameters can be reduced to two types of critical values: critical booking capacity and critical decision periods. These values play an important role in reducing the computational time and eliminating the need for a large amount of data storage. In an extension of Gerchak's work, Lee, and Hersh [10] developed a dynamic model with multiple fare classes and multiple seat bookings.

To generate the largest possible revenues, it is reasonable for airlines to offer unbooked seats in the business cabin to passengers who requested seats in the economy cabin, at no additional cost to the passengers. However, this was not taken into consideration in the models of the aforementioned literature. In the present paper, a model for a flight with two cabins and multi-fare classes within each cabin is proposed. In our model, demands are also modeled as a stochastic process, and the booking policy also can be reduced to some set of critical values. The booking policy includes the following information: (1) which fare classes should be opened for sale within each cabin (i.e. whether to accept a request for a fare class in each cabin), and (2) whether to accept a request for a fare class in the economy cabin once the economy cabin reaches full capacity.

To that end, this paper has developed a discrete time dynamic programming model which leads to a decision rule for the problem involving two cabins and multiple fare classes within each cabin. The objective of this paper is to maximize the expected total revenue.

\section{Problem Description and Modeling Assumption}

Consider a single-flight-leg multiple-fare-class airline seat inventory control problem. Suppose that an airline has previously specified a set of allowable fare classes, $A^{j}=\left\{1,2, \cdots, L^{j}\right\}$, $j \in\{1,2\}$, for a economy cabin $(j=1)$ and business cabin $(j=2)$ in a flight. The purpose of the airline is to optimally sell tickets (i.e. at a price that provides the airline the largest revenues possible).

By considering the policy in which passengers in the economy cabin can be offered seats in business cabin without paying additional cost, this paper has attempted to develop a booking policy to achieve this purpose. The booking policy that was developed includes the following information:

1. whether to accept a request for a fare class within the economy cabin when there are seats available within that cabin.

2. whether to accept a request for a fare class within the economy cabin when there is no seat available in that cabin but there are seats available in the business cabin.

3. whether to accept a request for a fare class in the business cabin when there are seats available within that cabin.

For the modeling purpose, the total planning horizon has been divided into $T$ decision periods which is small enough that no more than one customer arrives per period. Also, the decision periods are numbered in reverse sequence, i.e. $t=1$ will refer to the final decision 
period, $t=2$ to the period before the final decision period, and so on. It has been observed that $t$ has also been used to represent the number of periods remaining. Moreover, in this paper, fare classes are classified into ordered types $\ell, \ell \in\left\{1,2, \cdots, L^{j}\right\}$, where the ticket price of the fare class 1 is the most expensive and $L^{j}$ is the least expensive within cabin $j$.

In this paper, cancellations, no-shows, and overbookings are not considered. Indeed, these assumptions are also assumed by other authors (e.g., Brumelle et al. [4], Curry [6], Lee et al. [10], Robinson [12]). Furthermore, Brumelle et al. [4] have discussed the limitations. We agree with them that the analysis of this simplified version can serve as a basis for approximate solutions to more realistic versions. Finally, we assume that each passenger requests a single seat.

\section{The Model}

In this section, it is assumed that every request is only for one seat. Moreover, the following notation and functions are used.

Notation:

$j$ : the type of cabin; economy cabin when $j=1$ and business cabin when $j=2$,

$i_{j}$ : the remaining seats available for cabin $j$, (initially, $i_{j}=I_{j}$ )

$B_{t}^{j}\left(i_{1}, i_{2}\right)$ : a set of the opened fare classes in cabin $j$ during period $t$ when $i_{1}$ and $i_{2}$ seats remain (For simplicity, the symbol $B_{t}^{j}$ is used to short for $B_{t}^{j}\left(i_{1}, i_{2}\right)$ ),

$L^{j}:$ the number of fare classes within cabin $j$,

$x_{\ell}^{j}$ : the expected revenue from selling a seat in fare class $\ell$ within cabin $j$,

$\lambda_{t \ell}^{j}$ : the probability of a customer's requesting fare class $\ell$ in cabin $j$ in period $t$.

Functions:

$v_{t}\left(i_{1}, i_{2}\right)$ : the maximum total expected revenue that can be generated within $t$ periods when there are $i_{1}$ and $i_{2}$ seats remaining.

$v_{t}\left(i_{1}, i_{2}, B_{t}^{1}, B_{t}^{2}\right):$ the maximum total expected revenue within $t$ periods when there are $i_{1}$ and $i_{2}$ seats remaining, and the fare classes $B_{t}^{1}$ in cabin 1 and $B_{t}^{2}$ in cabin 2 are, respectively, opened for sale.

If there is no time remaining or no seat remaining for booking, no additional revenue can be generated. Therefore, $v_{t}(0,0)=0$ and $v_{0}\left(i_{1}, i_{2}\right)=0$. Moreover, during the booking periods, the following situations may arise: (1) there is no seat available for cabin 1 and cabin $2 ;(2)$ there are seats remaining in cabin 1 , but, no seat remaining in cabin $2 ;(3)$ there is no seat remaining in cabin 1 while there are seats remaining in cabin 2 ; and (4) there are seats remaining in both cabins 1 and 2 .

In case (1), all the fare classes in cabin 1 and cabin 2 should be closed (i.e. $B_{t}^{j}=\phi$, $j=1,2$ ), and so the total expected revenue is 0 ; in case (2), no fare class in cabin 2 will be opened for sale (i.e. $\left.B_{t}^{2}=\phi\right)$. Thus, the total expected revenues from selling $i_{1}$ seats within $t$ periods is given by $\left(1-\sum_{\ell \in B_{t}^{1}} \lambda_{t \ell}^{1}\right) v_{t-1}\left(i_{1}, 0\right)+\sum_{\ell \in B_{t}^{1}} \lambda_{t \ell}^{1}\left(x_{\ell}^{1}+v_{t-1}\left(i_{1}-1,0\right)\right)$. In case (3), since the free upgrading policy is used, a requests for cabin 1 can be upgraded for free to a seat in cabin 2 . Thus, the total expected revenue is $\left(1-\sum_{\ell \in B_{t}^{1}} \lambda_{t \ell}^{1}-\sum_{\ell \in B_{t}^{2}} \lambda_{t \ell}^{2}\right) v_{t-1}\left(0, i_{2}\right)+$ $\sum_{\ell \in B_{t}^{1}} \lambda_{t \ell}^{1}\left(x_{\ell}^{1}+v_{t-1}\left(0, i_{2}-1\right)\right)+\sum_{\ell \in B_{t}^{2}} \lambda_{t \ell}^{2}\left(x_{\ell}^{2}+v_{t-1}\left(0, i_{2}-1\right)\right)$. In case $(4)$, the fare classes $B_{t}^{1}$ and $B_{t}^{2}$ will be chosen for sale from $A^{1}$ and $A^{2}$, respectively. Thus, the total expected revenue is $\left(1-\sum_{\ell \in B_{t}^{1}} \lambda_{t \ell}^{1}-\sum_{\ell \in B_{t}^{2}} \lambda_{t \ell}^{2}\right) v_{t-1}\left(i_{1}, i_{2}\right)+\sum_{\ell \in B_{t}^{1}} \lambda_{t \ell}^{1}\left(x_{\ell}^{1}+v_{t-1}\left(i_{1}-1,0\right)\right)+\sum_{\ell \in B_{t}^{2}} \lambda_{t \ell}^{2}\left(x_{\ell}^{2}+\right.$ $\left.v_{t-1}\left(i_{1}, i_{2}-1\right)\right)$. Therefore, $v_{t}\left(i_{1}, i_{2}, B_{t}^{1}, B_{t}^{2}\right)$ is expressed as the following backward recursive equation: 


$$
\begin{aligned}
v_{t}\left(i_{1}, i_{2}, B_{t}^{1}, B_{t}^{2}\right)= & \left(1-\sum_{\ell \in B_{t}^{1}} \lambda_{t \ell}^{1} I\left(i_{1}=0, i_{2}=0\right)-\sum_{\ell \in B_{t}^{2}} \lambda_{t \ell}^{2} I\left(i_{2}=0\right)\right) v_{t-1}\left(i_{1}, i_{2}\right) \\
& +\sum_{\ell \in B_{t}^{1}} \lambda_{t \ell}^{1}\left(x_{\ell}^{1}+v_{t-1}\left(i_{1}-1, i_{2}\right)\right) I\left(i_{1} \geq 1\right) \\
& +\sum_{\ell \in B_{t}^{1}} \lambda_{t \ell}^{1}\left(x_{\ell}^{1}+v_{t-1}\left(0, i_{2}-1\right)\right) I\left(i_{1}=0, i_{2} \geq 1\right) \\
& +\sum_{\ell \in B_{t}^{2}} \lambda_{t \ell}^{2}\left(x_{\ell}^{2}+v_{t-1}\left(i_{1}, i_{2}-1\right)\right) I\left(i_{2} \geq 1\right)
\end{aligned}
$$

where $I(\cdot)$ is an indicator function with $I(S)=1$ if statement $S$ is true, or else $I(S)=0$. Since airlines will try to maximize the total expected revenue, $v_{t}\left(i_{1}, i_{2}\right)$ is expressed as follows:

$$
v_{t}\left(i_{1}, i_{2}\right)=\max _{B_{t}^{1} \subset A^{1}, B_{t}^{2} \subset A^{2}} v_{t}\left(i_{1}, i_{2}, B_{t}^{1}, B_{t}^{2}\right)
$$

Let $z_{t}^{j}\left(i_{1}, i_{2}\right)$ be the average maximum revenue per seat sold if the $i_{j}^{\prime}$ th seat is sold in period $t$, that is

$$
\begin{array}{lll}
z_{t}^{1}\left(i_{1}, i_{2}\right)=v_{t}\left(i_{1}, i_{2}\right)-v_{t}\left(i_{1}-1, i_{2}\right) & \text { for } & i_{1} \geq 1 \\
z_{t}^{2}\left(i_{1}, i_{2}\right)=v_{t}\left(i_{1}, i_{2}\right)-v_{t}\left(i_{1}, i_{2}-1\right) & \text { for } & i_{2} \geq 1
\end{array}
$$

Then, we can write $v_{t}\left(i_{1}, i_{2}\right)$ in $(2)$ in the following form

$$
\begin{aligned}
v_{t}\left(i_{1}, i_{2}\right)= & v_{t-1}\left(i_{1}, i_{2}\right)+K_{t}^{1}\left(z_{t-1}^{1}\left(i_{1}, i_{2}\right)\right) I\left(i_{1} \geq 1\right)+K_{t}^{1}\left(z_{t-1}^{2}\left(i_{1}, i_{2}\right)\right) I\left(i_{1}=0, i_{2} \geq 1\right) \\
& +K_{t}^{2}\left(z_{t-1}^{2}\left(i_{1}, i_{2}\right)\right) I\left(i_{2} \geq 1\right)
\end{aligned}
$$

where

$$
K_{t}^{j}(\nu)=\max _{B_{t}^{j} \subset A^{j}} \sum_{\ell \in B_{t}^{j}} \lambda_{t \ell}^{j}\left(x_{\ell}^{j}-\nu\right) .
$$

For any given $t, i_{1}$, and $i_{2}$, define $m_{t}^{j}\left(i_{1}, i_{2}\right)$ as the largest index of the opened booking class set $B_{t}^{j}$. That is,

$$
m_{t}^{j}\left(i_{1}, i_{2}\right)=\max _{\ell: \ell \in B_{t}^{j}} \ell
$$

Fare classes are ordered according to descending fare value to the airline. Thus, $m_{t}^{j}\left(i_{1}, i_{2}\right)$ can be interpreted as the least expensive fare class among the opened fare class in cabin $\mathrm{j}$ in period $t$ when $i_{1}$ and $i_{2}$ seats are available. Accordingly, the optimal opened booking classes is the set $B_{t}^{j}=\left\{1,2, \cdots, m_{t}^{j}\left(i_{1}, i_{2}\right)\right\}$. Therefore, if the index $m_{t}^{j}\left(i_{1}, i_{2}\right)$ is determined, the optimal booking class set $B_{t}^{j}$ is also determined. By (5) and (6), it is easy to determine the index, $m_{t}^{j}\left(i_{1}, i_{2}\right)$ and $B_{t}^{j}$, and we have the following theorem.

Theorem $3.1 m_{t}^{j}\left(i_{1}, i_{2}\right)=\max _{\ell: x_{\ell}^{j} \geq z_{t-1}^{j}\left(i_{1}, i_{2}\right), \ell \in A^{j}} \ell$.

The index, $m_{t}^{j}\left(i_{1}, i_{2}\right)$, can be obtained by directly computing (3) (5), and its application can be interpreted as follows. For example, if a customer who requests booking class $\ell$ in cabin $j$ arrives in period $t$ with $i_{1}$ and $i_{2}$ seats available, the request should be accepted if and only if $\ell \leq m_{t}^{j}\left(i_{1}, i_{2}\right)$. Thus, instead of storing the set $B_{t}^{j}\left(i_{1}, i_{2}\right)$, the airline can only store the value $m_{t}^{j}\left(i_{1}, i_{2}\right)$ for each different combination of $i_{1}, i_{2}$ and $t$ for each cabin $j$. 
It should be noted that for any given $j, m_{t}^{j}\left(i_{1}, i_{2}\right)$ is dependent on the values $i_{1}, i_{2}$, and $t$. Thus, the number of data storage for the booking policy for cabin $j$ is the value $I_{1} \times I_{2} \times T$.

If we can find some approaches to eliminate the unnecessary data storage, the booking system will be more efficient. An efficient method to achieve this result is the monotone control approach. By applying this approach, Gerchak et al. [6] control a two-fare class production problem with a few controlling data (the number of original data is the product of total decision periods and the total number of the booking capacities. Using the critical booking period, the number of the data storage is reduced to the product of the total booking capacities and the number of booking classes). An extension of Gerchak's model, Lee et al.[9] show that the multiple fare class airline booking problem also can be controlled by using either a set of critical booking periods or a set of critical booking capacities. This paper will show that the monotone control approach can also be applied in the case concerning a two-cabins multi-fare class airline seat inventory problem. To show this the following lemmas and theorems are needed:

Lemma 3.1 For any real numbers $\nu_{1}$ and $\nu_{2}$ such that $\nu_{1} \leq \nu_{2}$,

$$
0 \leq K_{t}^{j}\left(\nu_{1}\right)-K_{t}^{j}\left(\nu_{2}\right) \leq \sum_{\ell \in A^{j}} \lambda_{t \ell}^{j}\left(\nu_{2}-\nu_{1}\right)
$$

Proof: By (6) we have

$$
\begin{aligned}
K_{t}^{j}\left(\nu_{1}\right)-K_{t}^{j}\left(\nu_{2}\right) & =\max _{B_{t}^{j} \subset A^{j}} \sum_{\ell \in B_{t}^{j}} \lambda_{t \ell}^{j}\left(x_{\ell}-\nu_{1}\right)-\max _{B_{t}^{j} \subset A^{j}} \sum_{\ell \in B_{t}^{j}} \lambda_{t \ell}^{j}\left(x_{\ell}-\nu_{2}\right) \\
& \leq \max _{B_{t}^{j} \subset A^{j}} \sum_{\ell \in B_{t}^{j}} \lambda_{t \ell}^{j}\left(\nu_{2}-\nu_{1}\right)=\sum_{\ell \in A^{j}} \lambda_{t \ell}^{j}\left(\nu_{2}-\nu_{1}\right), \\
K_{t}^{j}\left(\nu_{1}\right)-K_{t}^{j}\left(\nu_{2}\right) & =\max _{B_{t}^{j} \subset A^{j}} \sum_{\ell \in B_{t}^{j}} \lambda_{t \ell}^{j}\left(x_{\ell}-\nu_{1}\right)-\max _{B_{t}^{j} \subset A^{j}} \sum_{\ell \in B_{t}^{j}} \lambda_{t \ell}^{j}\left(x_{\ell}-\nu_{2}\right) \\
& \geq \min _{B_{t}^{j} \subset A^{j}} \sum_{\ell \in B_{t}^{j}} \lambda_{t \ell}^{j}\left(\nu_{2}-\nu_{1}\right)=0 .
\end{aligned}
$$

Lemma 3.2 Let $B(\nu)$ be a set such that

$$
K(\nu)=\max _{B \in A} \sum_{\ell \in B} \lambda_{\ell}\left(x_{\ell}-\nu\right)=\sum_{\ell \in B(\nu)} \lambda_{\ell}\left(x_{\ell}-\nu\right) .
$$

Then, for any real numbers $\nu_{1}, \nu_{2}, \nu_{3}$, and $\nu_{4}$, the equation $q=K\left(\nu_{1}\right)-K\left(\nu_{2}\right)-K\left(\nu_{3}\right)+K\left(\nu_{4}\right)$ has the following properties:

(a) if $\nu_{2} \leq \min \left\{\nu_{1}, \nu_{4}\right\}$ and $\nu_{3} \geq \max \left\{\nu_{1}, \nu_{4}\right\}$, then $q \leq \sum_{\ell \in B\left(\nu_{1}\right)} \lambda_{\ell}\left(\nu_{2}-\nu_{1}+\nu_{3}-\nu_{4}\right)$,

(b) if $\nu_{1} \leq \min \left\{\nu_{2}, \nu_{3}\right\}$ and $\nu_{4} \geq \max \left\{\nu_{2}, \nu_{3}\right\}$, then $q \geq \sum_{\ell \in B\left(\nu_{2}\right)} \lambda_{\ell}\left(\nu_{2}-\nu_{1}+\nu_{3}-\nu_{4}\right)$.

Proof: (a) There are two possible cases.: Case (1) $\nu_{1} \leq \nu_{4}$. In this case, we have $\nu_{2} \leq \nu_{1} \leq$ $\nu_{4} \leq \nu_{3}$ and $B\left(\nu_{3}\right) \subseteq B\left(\nu_{4}\right) \subseteq B\left(\nu_{1}\right) \subseteq B\left(\nu_{2}\right)$, thus

$$
\begin{aligned}
q & =K\left(\nu_{1}\right)-K\left(\nu_{2}\right)-K\left(\nu_{3}\right)+K\left(\nu_{4}\right) \\
& =\sum_{\ell \in B\left(\nu_{1}\right)} \lambda_{\ell}\left(x_{\ell}-\nu_{1}\right)-\sum_{\ell \in B\left(\nu_{2}\right)} \lambda_{\ell}\left(x_{\ell}-\nu_{2}\right)+\sum_{\ell \in B\left(\nu_{4}\right)} \lambda_{\ell}\left(x_{\ell}-\nu_{4}\right)-\sum_{\ell \in B\left(\nu_{3}\right)} \lambda_{\ell}\left(x_{\ell}-\nu_{3}\right) \\
& \leq \sum_{\ell \in B\left(\nu_{1}\right)} \lambda_{\ell}\left(\nu_{2}-\nu_{1}\right)+\sum_{\ell \in B\left(\nu_{4}\right)} \lambda_{\ell}\left(\nu_{3}-\nu_{4}\right) \leq \sum_{\ell \in B\left(\nu_{1}\right)} \lambda_{\ell}\left(\nu_{2}-\nu_{1}\right)+\sum_{\ell \in B\left(\nu_{1}\right)} \lambda_{\ell}\left(\nu_{3}-\nu_{4}\right) \\
& \leq \sum_{\ell \in B\left(\nu_{1}\right)} \lambda_{\ell}\left(\nu_{2}-\nu_{1}+\nu_{3}-\nu_{4}\right) .
\end{aligned}
$$

Case (2) $\nu_{1} \geq \nu_{4}$. The proof is similar to case (1).

(b) The proof of (b) is almost the same as the proof of (a). 
Lemma $3.3 z_{t}^{1}\left(i_{1}, i_{2}\right)$ and $z_{t}^{2}\left(i_{1}, i_{2}\right)$ are nonincreasing in $i_{2}$ and $i_{1}$, respectively.

Proof: See Appendix.

Lemma $3.4 v_{t}\left(i_{1}, i_{2}-1\right) \leq v_{t}\left(i_{1}-1, i_{2}\right)$.

Proof: Since a seat within cabin 2 can be sold to a customer who requests a seat within cabin 1 , while, a seat within cabin 1 can not be sold to a customer who requests a seat in cabin 2 .

Lemma 3.5 Suppose $z_{t}^{j}\left(i_{1}, i_{2}\right)$ is nonincreasing in $i_{j}$. Then, $F_{t}\left(i_{1}, i_{2}\right)=v_{t}\left(i_{1}, i_{2}\right)-v_{t}\left(i_{1}-\right.$ $\left.1, i_{2}+1\right)$ is nonincreasing in $i_{1}$ and nondecreasing in $i_{2}$.

Proof: See Appendix.

Combining the above lemmas, one obtains the following theorem.

Theorem $3.2 z_{t}^{j}\left(i_{1}, i_{2}\right)$ is nonincreasing in $i_{j}$.

Proof: The proof again will be given in the appendix.

Similarly, one can also prove the following theorem.

Theorem $3.3 z_{t}^{j}\left(i_{1}, i_{2}\right)$ is nondecreasing in $t$.

Proof: See Appendix.

\subsection{Critical Booking Capacities}

In this subsection, we will show that the booking process can be controlled by using some critical values.

Theorem $3.4 m_{t}^{j}\left(i_{1}, i_{2}\right)$ is nondecreasing in $i_{1}$ and $i_{2}$.

Proof: Immediate from Theorem 3.1 and Theorem 3.2.

The decisions of the proposed problem are represented by the set $B_{t}^{j}=\left\{1,2, \cdots, m_{t}^{j}\left(i_{1}, i_{2}\right)\right\}$ for each different combination of $i_{1}, i_{2}$ and $t$. Thus, a request for booking class $\ell$ in cabin $\mathrm{j}$ with $i_{1}$ and $i_{2}$ seats available should be accepted if and only if $\ell \leq m_{t}^{j}\left(i_{1}, i_{2}\right)$. The value $m_{t}^{j}\left(i_{1}, i_{2}\right)$ is dependent on $t, i_{1}, i_{2}$, and $j$, thus the number of the data storage for cabin $\mathrm{j}$ is $T \times I_{1} \times I_{2}$.

In general, the values $I_{1}$ and $I_{2}$ are several times more than the total booking classes $L^{j}$. Accordingly, $T \times I_{1} \times I_{2}$ is several times more than $I_{2} \times T \times L^{j}$. Below, we show that the number of original data can be reduced to the number $I_{2} \times T \times L^{j}$.

Since $m_{t}^{j}\left(i_{1}, i_{2}\right)$ is nondecreasing in $i_{1}$ and $i_{2}$, there exists some critical booking capacity, $i_{1}\left(\ell, t, i_{2}\right)\left(i_{2}\left(\ell, t, i_{1}\right)\right)$, such that $\ell \leq m_{t}^{1}\left(i_{1}, i_{2}\right)\left(\ell \leq m_{t}^{2}\left(i_{1}, i_{2}\right)\right)$ if and only if $i_{1} \geq i_{1}\left(\ell, t, i_{2}\right)$ $\left(i_{2} \geq i_{2}\left(\ell, t, i_{1}\right)\right)$ (Figure 1$)$.

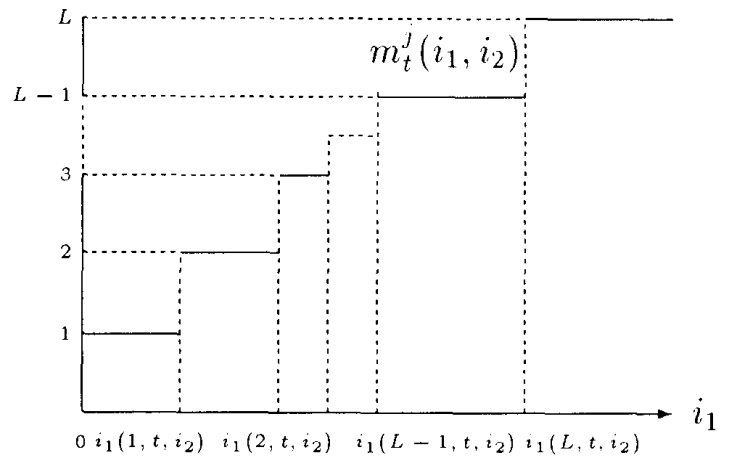

Figure 1: Critical booking capacity

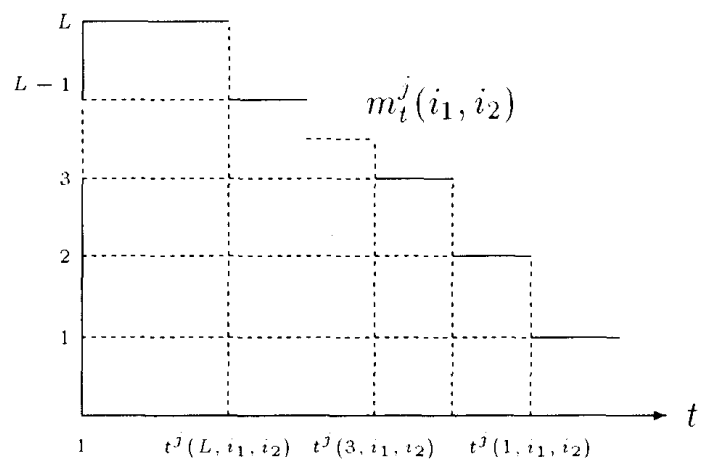

Figure 2: Critical decision period 
Therefore, the booking policy can be expressed as follows:

- The Booking Policy using critical booking capacity

1. for given $t$ and $i_{1} \geq 1$, a request for booking class $\ell$ in cabin 1 should be accepted if and only if $i_{1} \geq i_{1}\left(\ell, t, i_{2}\right)$.

2. for given $t$ and $i_{1}=0$, a request for booking class $\ell$ in cabin 1 should be upgraded for free to a seat in business cabin if and only if $i_{2} \geq i_{2}\left(\ell, t, i_{1}\right)$.

3 . for given $t$ and $i_{2} \geq 0$, a request for booking class $\ell$ in cabin 2 should be accepted if and only if $i_{2} \geq i_{2}\left(\ell, t, i_{1}\right)$.

It is noted that $i_{1}\left(\ell, t, i_{2}\right)$ is the minimum $i_{1}$ among the index set $\left\{i_{1}: m_{t}^{1}\left(i_{1}, i_{2}\right)=\ell\right\}$ and $m_{t}^{1}\left(i_{1}, i_{2}\right)$ is nondecreasing in $i_{1}$ from Theorm 3.4. Thus, $i_{1}\left(\ell, t, i_{2}\right)$ is nondecreasing in $\ell$. Similarly, $i_{2}\left(\ell, t, i_{1}\right)$ is nondecreasing in $\ell$.

\subsection{Critical Booking Periods}

Theorem $3.5 m_{t}^{j}\left(i_{1}, i_{2}\right)$ is nonincreasing in $t$.

Proof: Immediate from Theorm 3.3.

In this section, we use the critical booking periods to reduce the data storage. Here, the number of the data storage for cabin $\mathrm{j}$ is reduced to $I_{1} \times I_{2} \times L^{j}$.

Since $m_{t}^{j}\left(i_{1}, i_{2}\right)$ is nonincreasing in $t$, there exists some critical booking period, $t^{1}\left(\ell, i_{1}, i_{2}\right)$ $\left(t^{2}\left(\ell, i_{1}, i_{2}\right)\right)$, such that $\ell \leq m_{t}^{1}\left(i_{1}, i_{2}\right)\left(\ell \leq m_{t}^{2}\left(i_{1}, i_{2}\right)\right)$ if and only if $t \leq t^{1}\left(\ell, i_{1}, i_{2}\right)(t \leq$ $\left.t^{2}\left(\ell, i_{1}, i_{2}\right)\right)$ (Figure 2). Thus, the following booking strategy can be used to control the booking process.

- The Booking Policy using critical booking period

1. for given $i_{1} \geq 1$ and $i_{2}$, a request of booking class $\ell$ in cabin 1 should be accepted if and only if $t \leq t^{1}\left(\ell, i_{1}, i_{2}\right)$.

2. for given $i_{1}=0$ and $i_{2} \geq 1$, a request of booking class $\ell$ in cabin 1 should be upgraded for free to a seat in business cabin if and only if $t \leq t^{1}\left(\ell, 0, i_{2}\right)$.

3. for given $i_{1} \geq 1$ and $i_{2} \geq 0$, a request of booking class $\ell$ in cabin 2 should be accepted if and only if $t \leq t^{2}\left(\ell, i_{1}, i_{2}\right)$.

It is noted that $t^{j}\left(\ell, i_{1}, i_{2}\right)$ is the maximum $t$ among the index set $\left\{t: m_{t}^{j}\left(i_{1}, i_{2}\right)=\ell\right\}$ and $m_{t}^{j}\left(i_{1}, i_{2}\right)$ is nonincreasing in $t$ from Theorm 3.5. Thus, $t^{j}\left(\ell, i_{1}, i_{2}\right)$ is nondecreasing in $\ell$. Now, we will describe how to search the critical values. Observe that for given $\ell, t$, and $i_{2}$, $i_{1}\left(\ell, t, i_{2}\right)$ is the minimum $i_{1}$ among the index set $\left\{i_{1}: m_{t}^{1}\left(i_{1}, i_{2}\right)=\ell\right\}$. Therefore, by Theorm 3.1 and Theorm 3.2 , the value $i_{1}\left(\ell, t, i_{2}\right)$ can be obtained from the following equation:

$$
i_{1}\left(\ell, t, i_{2}\right)=\min \left\{i_{1}: x_{\ell}^{1} \geq z_{t-1}^{\mathrm{l}}\left(i_{1}, i_{2}\right)\right\}
$$

Similarly, we have

$$
i_{2}\left(\ell, t, i_{1}\right)=\min \left\{i_{2}: x_{\ell}^{2} \geq z_{t-1}^{2}\left(i_{1}, i_{2}\right)\right\}
$$

Moreover, by Theorm 3.1 and Theorm 3.3, the critical booking periods are given by

$$
\begin{aligned}
& t^{1}\left(\ell, i_{1}, i_{2}\right)=\max \left\{t: x_{\ell}^{1} \geq z_{t-1}^{1}\left(i_{1}, i_{2}\right)\right\}, \\
& t^{2}\left(\ell, i_{1}, i_{2}\right)=\max \left\{t: x_{\ell}^{2} \geq z_{t-1}^{2}\left(i_{1}, i_{2}\right)\right\} .
\end{aligned}
$$




\section{Numerical Example}

In order to illustrate the proposed model and the booking policies, an example is described as follows. Assume a flight will be departing after $T=400$ planning periods. The maximum booking capacity for the economy cabin and the business cabin of the flight are $I_{1}=100$ and $I_{2}=50$, respectively. Also, assume that the airline has previously specified $L=4$ fare classes for both cabins and their corresponding ticket price $x_{\ell}^{j}$ and the arriving probabilities $\lambda_{t}^{\ell}$ are as in Table I and Table II, respectively.

Table I: the expected

\begin{tabular}{|c|c|c|c|c|}
\hline \multirow[b]{2}{*}{$j$} & \multicolumn{3}{|c|}{$\ell$} & \multirow[b]{2}{*}{4} \\
\hline & 1 & 2 & 3 & \\
\hline 1 & 300 & 200 & 100 & 50 \\
\hline 2 & 500 & 400 & 350 & 300 \\
\hline
\end{tabular}

Table II: Request Probabilities $\lambda_{t \ell}^{j}$

\begin{tabular}{|c|c|c|c|c|c|c|c|c|}
\hline \multirow[b]{2}{*}{$t$} & \multicolumn{4}{|c|}{$j=1$} & \multicolumn{4}{|c|}{$j=2$} \\
\hline & $1: 100$ & $101: 200$ & $201: 300$ & $301: 400$ & $1: 100$ & 101:200 & $201: 300$ & $301: 400$ \\
\hline$\lambda_{t 1}^{j}$ & 0.08 & 0.07 & 0.06 & 0.03 & 0.08 & 0.06 & 0.04 & 0.03 \\
\hline$\lambda_{t 2}^{j}$ & 0.09 & 0.05 & 0.02 & 0.03 & 0.05 & 0.04 & 0.03 & 0.03 \\
\hline$\lambda_{t 3}^{j}$ & 0.06 & 0.07 & 0.05 & 0.03 & 0.05 & 0.07 & 0.03 & 0.02 \\
\hline$\lambda_{t 4}^{J}$ & 0.03 & 0.02 & 0.05 & 0.06 & 0.02 & 0.03 & 0.04 & 0.03 \\
\hline
\end{tabular}

Tables III-X show some values of the critical booking periods. The application of these Tables can be interpreted as follows. For example, from Tables III-VI, if there are $i_{1}=40$, $i_{2}=20$ seats on hand, a request for fare classes $1,2,3$ and 4 in cabin 1 is accepted if and only if his/her arriving time is $t \leq 400, t \leq 400, t \leq 238$, and $t \leq 168$, respectively. From Tables III-VI, if $i_{1}=0$ and $i_{2}=10$, a request for fare classes 1, 2, 3 and 4 in cabin 1 should be upgraded for free to a seat in business cabin if and only if his/her arriving time is $t \leq 50$, $t \leq 31, t \leq 22$, and $t \leq 18$, respectively.

From Table VII-X, if there are $i_{1}=40, i_{2}=20$ seats on hand, a request for fare classes $1,2,3$ and 4 in cabin 1 is accepted if and only if his/her arriving time is $t \leq 400, t \leq 120$, $t \leq 86$, and $t \leq 70$, respectively.

Not only the critical booking period but also the critical booking capacity can be used in controlling the booking process. In this example, since the total number of periods is several times more than the total booking capacities and the number of booking classes, using the critical booking period to control the booking process is more efficient than using the critical booking capacity.

Table III Critical booking periods for $L=1$ in cabin 1

\begin{tabular}{ccccccc}
\hline & \multicolumn{7}{c}{$i_{2}$} \\
\cline { 2 - 7 }$i_{1}$ & 0 & 10 & 20 & 30 & 40 & 50 \\
\hline 0 & 0 & 50 & 98 & 150 & 203 & 269 \\
20 & 400 & 400 & 400 & 400 & 400 & 400 \\
40 & 400 & 400 & 400 & 400 & 400 & 400 \\
60 & 400 & 400 & 400 & 400 & 400 & 400 \\
80 & 400 & 400 & 400 & 400 & 400 & 400 \\
100 & 400 & 400 & 400 & 400 & 400 & 400 \\
\hline
\end{tabular}

Table IV Critical booking periods for $L=2$ in cabin 1

\begin{tabular}{ccccccc}
\hline & \multicolumn{7}{c}{$i_{2}$} \\
\cline { 2 - 7 }$i_{1}$ & 0 & 10 & 20 & 30 & 40 & 50 \\
\hline 0 & 0 & 31 & 62 & 93 & 126 & 160 \\
20 & 192 & 192 & 193 & 196 & 210 & 247 \\
40 & 400 & 400 & 400 & 400 & 400 & 400 \\
60 & 400 & 400 & 400 & 400 & 400 & 400 \\
80 & 400 & 400 & 400 & 400 & 400 & 400 \\
100 & 400 & 400 & 400 & 400 & 400 & 400 \\
\hline
\end{tabular}


Table V Critical booking periods for $L=3$ in cabin 1

\begin{tabular}{ccccccc}
\hline & \multicolumn{7}{c}{$i_{2}$} \\
\cline { 2 - 7 }$i_{1}$ & 0 & 10 & 20 & 30 & 40 & 50 \\
\hline 0 & 0 & 22 & 46 & 71 & 96 & 123 \\
20 & 96 & 97 & 102 & 124 & 151 & 179 \\
40 & 237 & 237 & 238 & 240 & 244 & 263 \\
60 & 400 & 400 & 400 & 400 & 400 & 400 \\
80 & 400 & 400 & 400 & 400 & 400 & 400 \\
100 & 400 & 400 & 400 & 400 & 400 & 400 \\
\hline
\end{tabular}

Table VII Critical booking periods for $L=1$ in cabin 2

\begin{tabular}{cccccc}
\hline & \multicolumn{5}{c}{$i_{2}$} \\
\cline { 2 - 6 }$i_{1}$ & 10 & 20 & 30 & 40 & 50 \\
\hline 0 & 400 & 400 & 400 & 400 & 400 \\
20 & 400 & 400 & 400 & 400 & 400 \\
40 & 400 & 400 & 400 & 400 & 400 \\
60 & 400 & 400 & 400 & 400 & 400 \\
80 & 400 & 400 & 400 & 400 & 400 \\
100 & 400 & 400 & 400 & 400 & 400 \\
\hline
\end{tabular}

Table IX Critical booking periods for $L=3$ in cabin 2

\begin{tabular}{cccccc}
\hline & \multicolumn{5}{c}{$i_{2}$} \\
\cline { 2 - 6 }$i_{1}$ & 10 & 20 & 30 & 40 & 50 \\
\hline 0 & 7 & 79 & 161 & 264 & 392 \\
20 & 9 & 85 & 167 & 270 & 396 \\
40 & 9 & 86 & 172 & 276 & 400 \\
60 & 9 & 86 & 173 & 279 & 400 \\
80 & 9 & 86 & 173 & 281 & 400 \\
100 & 9 & 86 & 173 & 281 & 400 \\
\hline
\end{tabular}

Table VI Critical booking periods for $L=4$ in cabin 1

\begin{tabular}{ccccccc}
\hline & \multicolumn{7}{c}{$i_{2}$} \\
\cline { 2 - 7 }$i_{1}$ & 0 & 10 & 20 & 30 & 40 & 50 \\
\hline 0 & 0 & 18 & 39 & 61 & 83 & 106 \\
20 & 74 & 75 & 85 & 106 & 131 & 155 \\
40 & 166 & 167 & 168 & 170 & 184 & 208 \\
60 & 287 & 287 & 287 & 288 & 289 & 295 \\
80 & 400 & 400 & 400 & 400 & 400 & 400 \\
100 & 400 & 400 & 400 & 400 & 400 & 400 \\
\hline
\end{tabular}

Table VIII Critical booking periods for $L=2$ in cabin 2

\begin{tabular}{cccccc}
\hline & \multicolumn{5}{c}{$i_{2}$} \\
\cline { 2 - 6 }$i_{1}$ & 10 & 20 & 30 & 40 & 50 \\
\hline 0 & 12 & 114 & 261 & 400 & 400 \\
20 & 13 & 118 & 264 & 400 & 400 \\
40 & 13 & 120 & 268 & 400 & 400 \\
60 & 13 & 120 & 270 & 400 & 400 \\
80 & 13 & 120 & 271 & 400 & 400 \\
100 & 13 & 120 & 271 & 400 & 400 \\
\hline
\end{tabular}

Table X Critical booking periods for $L=4$ in cabin 1

\begin{tabular}{cccccc}
\hline & \multicolumn{5}{c}{$i_{2}$} \\
\cline { 2 - 6 }$i_{1}$ & 10 & 20 & 30 & 40 & 50 \\
\hline 0 & 5 & 55 & 103 & 155 & 209 \\
20 & 6 & 69 & 128 & 187 & 260 \\
40 & 6 & 70 & 133 & 194 & 272 \\
60 & 6 & 70 & 134 & 198 & 276 \\
80 & 6 & 70 & 134 & 198 & 279 \\
100 & 6 & 70 & 134 & 198 & 279 \\
\hline
\end{tabular}

\section{Conclusion}

This paper studied a seat inventory problem in the case of multiple-fare classes and two cabins on a single-flight leg. In many previous models, a seat in business cabin can not be sold to a customer who requests economy cabin. However, in reality, a customer who requests economy seat may be offered a seat in the business cabin at no additional cost. Taking this fact into account, this paper proposed a single-flight-leg multi-fare class seat inventory control model.

We characterize the booking policy as follows: the booking policy can be controlled using either a set of critical booking capacities (The Booking Policy using critical booking capacity), or a set of critical decision periods (The Booking Policy using critical booking period).

This paper allows the condition that customers of economy class may be upgraded to business class. The model can be extended to the models with multiple cabins, overbooking, no-show, go-show and cancellation. Such extensions would be worthy subjects for future research. 


\section{Acknowledgment}

The authors thank the referees for their valuable comments and suggestions, which helped to improve this paper. This research is supported in part by Grant NSC 89-2416-H-309-003-.

\section{Appendix:}

\section{Proof of Lemma 3.3}

It suffices to show that $z_{1}^{1}\left(i_{1}, i_{2}\right)-z_{1}^{1}\left(i_{1}, i_{2}-1\right) \leq 0$ since $z_{t}^{1}\left(i_{1}, i_{2}\right)-z_{t}^{1}\left(i_{1}, i_{2}-1\right)=$ $z_{t}^{2}\left(i_{1}, i_{2}\right)-z_{t}^{2}\left(i_{1}-1, i_{2}\right)$. First, we will note that

$$
z_{1}^{1}\left(i_{1}, i_{2}\right)-z_{1}^{1}\left(i_{1}, i_{2}-1\right)= \begin{cases}-K_{1}^{1}(0) \leq 0, & \text { if } i_{1}=1 \text { and } i_{2}=1 \\ 0, & \text { otherwise }\end{cases}
$$

Thus, the statement holds for $t=1$. Assume the statements hold for $t-1$. For $i_{1} \geq 1$ and $i_{2} \geq 1$ we have

$$
z_{t}^{1}\left(i_{1}, i_{2}\right)-z_{t}^{1}\left(i_{1}, i_{2}-1\right)=\sum_{k=1}^{9} G_{k}
$$

where

$$
\begin{aligned}
G_{1} & =z_{t-1}^{1}\left(i_{1}, i_{2}\right)-z_{t-1}^{1}\left(i_{1}, i_{2}-1\right) \leq 0 \\
G_{2} & =K_{t}^{1}\left(z_{t-1}^{1}\left(i_{1}, i_{2}\right)\right) \\
G_{3} & =K_{t}^{2}\left(z_{t-1}^{2}\left(i_{1}, i_{2}\right)\right) \\
G_{4} & =-K_{t}^{1}\left(z_{t-1}^{1}\left(i_{1}-1, i_{2}\right)\right) I\left(i_{1} \geq 2\right)-K_{t}^{1}\left(z_{t-1}^{2}\left(i_{1}-1, i_{2}\right)\right) I\left(i_{1}=1, i_{2} \geq 1\right) \\
G_{5} & =-K_{t}^{2}\left(z_{t-1}^{2}\left(i_{1}-1, i_{2}\right)\right) \\
G_{6} & =-K_{t}^{1}\left(z_{t-1}^{1}\left(i_{1}, i_{2}-1\right)\right) \\
G_{7} & =-K_{t}^{2}\left(z_{t-1}^{2}\left(i_{1}, i_{2}-1\right)\right) I\left(i_{2} \geq 2\right) \\
G_{8} & =K_{t}^{1}\left(z_{t-1}^{1}\left(i_{1}-1, i_{2}-1\right)\right) I\left(i_{1} \geq 2\right)+K_{t}^{1}\left(z_{t-1}^{2}\left(i_{1}-1, i_{2}-1\right)\right) I\left(i_{1}=1, i_{2} \geq 2\right) \\
G_{9} & =K_{t}^{2}\left(z_{t-1}^{2}\left(i_{1}-1, i_{2}-1\right)\right) I\left(i_{2} \geq 2\right)
\end{aligned}
$$

Here, by Lemma 3.1 we obtain

$$
\begin{aligned}
G_{4}+G_{8} & \leq 0 \\
G_{2}+G_{6} & \leq-\sum_{\ell \in A^{1}} \lambda_{t \ell}^{1} G_{1} \\
G_{3}+G_{5} & \leq \sum_{\ell \in A^{2}} \lambda_{t \ell}^{2}\left(z_{t-1}^{2}\left(i_{1}-1, i_{2}\right)-z_{t-1}^{2}\left(i_{1}, i_{2}\right)\right)=-\sum_{\ell \in A^{2}} \lambda_{t \ell}^{2} G_{1}, \\
G_{7}+G_{9} & \leq 0 .
\end{aligned}
$$

Thus, $z_{t}^{1}\left(i_{1}, i_{2}\right)-z_{t}^{1}\left(i_{1}, i_{2}-1\right)=\sum_{k=1}^{9} G_{k} \leq\left(1-\sum_{\ell \in A^{1}} \lambda_{t \ell}^{1}-\sum_{\ell \in A^{2}} \lambda_{t \ell}^{2}\right) G_{1} \leq 0$.

\section{Proof of Lemma 3.5}

From (5) we have

$$
\begin{aligned}
& F_{1}\left(i_{1}, i_{2}\right)-F_{1}\left(i_{1}-1, i_{2}\right)=0, \\
& F_{1}\left(i_{1}, i_{2}\right)-F_{1}\left(i_{1}, i_{2}-1\right)= \begin{cases}K_{1}^{2}(0) \geq 0, & \text { if } i_{2}=1, \\
0, & \text { otherwise. }\end{cases}
\end{aligned}
$$

Assume the statement holds for $t-1$. Then, well have 


$$
\begin{aligned}
F_{t}\left(i_{1}, i_{2}\right)- & F_{t}\left(i_{1}-1, i_{2}\right)=F_{t-1}\left(i_{1}, i_{2}\right)-F_{t-1}\left(i_{1}-1, i_{2}\right) \\
& +K_{t}^{1}\left(z_{t-1}^{1}\left(i_{1}, i_{2}\right)\right)-K_{t}^{1}\left(z_{t-1}^{1}\left(i_{1}-1, i_{2}+1\right)\right) \\
& +K_{t}^{1}\left(z_{t-1}^{1}\left(i_{1}-2, i_{2}+1\right)\right)-K_{t}^{1}\left(z_{t-1}^{1}\left(i_{1}-1, i_{2}\right)\right) \\
& +K_{t}^{2}\left(z_{t-1}^{2}\left(i_{1}, i_{2}\right)\right)-K_{t}^{2}\left(z_{t-1}^{2}\left(i_{1}-1, i_{2}+1\right)\right) \\
& +K_{t}^{2}\left(z_{t-1}^{2}\left(i_{1}-2, i_{2}+1\right)\right)-K_{t}^{2}\left(z_{t-1}^{2}\left(i_{1}-1, i_{2}\right)\right) .
\end{aligned}
$$

Since $z_{t-1}^{1}\left(i_{1}, i_{2}\right)-z_{t-1}^{1}\left(i_{1}-1, i_{2}+1\right)=F_{t-1}\left(i_{1}, i_{2}\right)-F_{t-1}\left(i_{1}-1, i_{2}\right) \leq 0$ and $z_{t-1}^{1}\left(i_{1}-2, i_{2}+\right.$ 1) $-z_{t-1}^{1}\left(i_{1}-1, i_{2}\right)=F_{t-1}\left(i_{1}-2, i_{2}\right)-F_{t-1}\left(i_{1}-1, i_{2}\right) \geq 0$, by Lemma 3.1 we have

$$
\begin{aligned}
K_{t}^{1}\left(z_{t-1}^{1}\left(i_{1}, i_{2}\right)\right) & -K_{t}^{1}\left(z_{t-1}^{1}\left(i_{1}-1, i_{2}+1\right)\right) \\
& \leq \sum_{\ell \in B_{t}^{1}} \lambda_{t \ell}^{1}\left(z_{t-1}^{1}\left(i_{1}-1, i_{2}+1\right)-z_{t-1}^{1}\left(i_{1}, i_{2}\right)\right) \\
& =-\sum_{\ell \in B_{t}^{1}} \lambda_{t \ell}^{1}\left(F_{t-1}\left(i_{1}, i_{2}\right)-F_{t-1}\left(i_{1}-1, i_{2}\right)\right), \\
K_{t}^{1}\left(z_{t-1}^{1}\left(i_{1}-2, i_{2}+1\right)\right) & -K_{t}^{1}\left(z_{t-1}^{1}\left(i_{1}-1, i_{2}\right)\right) \leq 0 .
\end{aligned}
$$

Note that

$$
z_{t-1}^{2}\left(i_{1}-1, i_{2}+1\right) \leq \min \left\{z_{t-1}^{2}\left(i_{1}, i_{2}\right), z_{t-1}^{2}\left(i_{1}-1, i_{2}\right)\right\}
$$

since $z_{t-1}^{2}\left(i_{1}-1, i_{2}+1\right)-z_{t-1}^{2}\left(i_{1}, i_{2}\right)=F_{t-1}\left(i_{1}, i_{2}-1\right)-F_{t-1}\left(i_{1}, i_{2}\right) \leq 0$ and $z_{t-1}^{2}\left(i_{1}-\right.$ $\left.1, i_{2}+1\right) \leq z_{t-1}^{2}\left(i_{1}-1, i_{2}\right)$ from the assumption of this lemma. Moreover, $z_{t-1}^{2}\left(i_{1}, i_{2}-1\right) \geq$ $\max \left\{z_{t-1}^{2}\left(i_{1}, i_{2}\right), z_{t-1}^{2}\left(i_{1}-1, i_{2}\right)\right\}$ since $z_{t-1}^{2}\left(i_{1}, i_{2}-1\right) \geq z_{t-1}^{2}\left(i_{1}, i_{2}\right)$ from the assumption of this lemma and $z_{t-1}^{2}\left(i_{1}, i_{2}-1\right)-z_{t-1}^{2}\left(i_{1}-1, i_{2}\right)=F_{t-1}\left(i_{1}, i_{2}-1\right)-F_{t-1}\left(i_{1}, i_{2}-2\right) \geq 0$. Thus, by Lemma $3.2(\mathrm{a})$,

$$
\begin{aligned}
& K_{t}^{2}\left(z_{t-1}^{2}\left(i_{1}, i_{2}\right)\right)-K_{t}^{2}\left(z_{t-1}^{2}\left(i_{1}-1, i_{2}+1\right)\right)+K_{t}^{2}\left(z_{t-1}^{2}\left(i_{1}-2, i_{2}+1\right)\right)-K_{t}^{2}\left(z_{t-1}^{2}\left(i_{1}-1, i_{2}\right)\right) \\
& \quad \leq \sum_{\ell \in B_{t}^{2}} \lambda_{t \ell}^{2}\left(z_{t-1}^{2}\left(i_{1}-1, i_{2}+1\right)-z_{t-1}^{2}\left(i_{1}, i_{2}\right)+z_{t-1}^{2}\left(i_{1}-1, i_{2}\right)-z_{t-1}^{2}\left(i_{1}-2, i_{2}+1\right)\right) \\
& \quad=\sum_{\ell \in B_{t}^{2}} \lambda_{t \ell}^{2}\left(F_{t-1}\left(i_{1}-1, i_{2}\right)-F_{t-1}\left(i_{1}, i_{2}\right)+F_{t-1}\left(i_{1}, i_{2}-1\right)-F_{t-1}\left(i_{1}-1, i_{2}-1\right)\right) \\
& \quad \leq \sum_{\ell \in B_{t}^{2}} \lambda_{t \ell}^{2}\left(F_{t-1}\left(i_{1}-1, i_{2}\right)-F_{t-1}\left(i_{1}, i_{2}\right)\right) .
\end{aligned}
$$

Substituting (35), (36) and (37) into (34), we obtain

$$
\begin{aligned}
& F_{t}\left(i_{1}, i_{2}\right)-F_{t}\left(i_{1}-1, i_{2}\right) \\
& \quad=\left(1-\sum_{\ell \in B_{t}^{1}} \lambda_{t \ell}^{1}-\sum_{\ell \in B_{t}^{2}} \lambda_{t \ell}^{2}\right)\left(F_{t-1}\left(i_{1}, i_{2}\right)-F_{t-1}\left(i_{1}-1, i_{2}\right)\right) \leq 0 .
\end{aligned}
$$

Using Lemma 3.2(b) and similar arguments as the above, one can show that $F_{t}\left(i_{1}, i_{2}\right)-$ $F_{t}\left(i_{1}, i_{2}-1\right) \geq 0$. Therefore, $F_{t}\left(i_{1}, i_{2}\right)$ is nonincreasing in $i_{1}$ and nondecreasing in $i_{2}$.

\section{Proof of Theorm 3.2}

First, we will note that

$$
\begin{aligned}
& z_{1}^{1}\left(i_{1}, i_{2}\right)-z_{1}^{1}\left(i_{1}-1, i_{2}\right)= \begin{cases}-K_{1}^{1}(0) \leq 0, & \text { if } i_{1}=2, \\
0, & \text { otherwise }\end{cases} \\
& z_{1}^{2}\left(i_{1}, i_{2}\right)-z_{1}^{2}\left(i_{1}, i_{2}-1\right)= \begin{cases}-K_{1}^{1}(0)-K_{1}^{2}(0) \leq 0, & \text { if } i_{2}=2, \\
0, & \text { otherwise }\end{cases}
\end{aligned}
$$


Assume the statements hold for $t-1$. For $i_{1} \geq 2$ and $i_{2} \geq 0$

where

$$
z_{t}^{1}\left(i_{1}, i_{2}\right)-z_{t}^{1}\left(i_{1}-1, i_{2}\right)=\sum_{k=1}^{9} H_{k}
$$

$$
\begin{aligned}
& H_{1}=z_{t-1}^{1}\left(i_{1}, i_{2}\right)-z_{t-1}^{1}\left(i_{1}-1, i_{2}\right) \leq 0 \\
& H_{2}=K_{t}^{1}\left(z_{t-1}^{1}\left(i_{1}, i_{2}\right)\right) \\
& H_{3}=K_{t}^{2}\left(z_{t-1}^{2}\left(i_{1}, i_{2}\right)\right) I\left(i_{2} \geq 1\right) \\
& H_{4}=-K_{t}^{1}\left(z_{t-1}^{1}\left(i_{1}-1, i_{2}\right)\right) \\
& H_{5}=-K_{t}^{2}\left(z_{t-1}^{2}\left(i_{1}-1, i_{2}\right)\right) I\left(i_{2} \geq 1\right) \\
& H_{6}=-K_{t}^{1}\left(z_{t-1}^{1}\left(i_{1}-1, i_{2}\right)\right) \\
& H_{7}=-K_{t}^{2}\left(z_{t-1}^{2}\left(i_{1}-1, i_{2}\right)\right) I\left(i_{2} \geq 1\right), \\
& H_{8}=K_{t}^{1}\left(z_{t-1}^{1}\left(i_{1}-2, i_{2}\right)\right) I\left(i_{1} \geq 3\right),+K_{t}^{1}\left(z_{t-1}^{2}\left(i_{1}-2, i_{2}\right)\right) I\left(i_{1}=2, i_{2} \geq 1\right), \\
& H_{9}=K_{t}^{2}\left(z_{t-1}^{2}\left(i_{1}-2, i_{2}\right)\right) I\left(i_{2} \geq 1\right) .
\end{aligned}
$$

Since $z_{t-1}^{1}\left(i_{1}-2, i_{2}\right) \geq z_{t-1}^{1}\left(i_{1}-1, i_{2}\right)$ and by Lemma 3.4

$$
\begin{aligned}
z_{t-1}^{2}\left(i_{1}-2, i_{2}\right) & =v_{t-1}\left(i_{1}-2, i_{2}\right)-v_{t-1}\left(i_{1}-2, i_{2}-1\right) \\
& \geq v_{t-1}\left(i_{1}-1, i_{2}-1\right)-v_{t-1}\left(i_{1}-2, i_{2}-1\right) \\
& =z_{t-1}^{1}\left(i_{1}-1, i_{2}-1\right) \geq z_{t-1}^{1}\left(i_{1}-1, i_{2}\right)
\end{aligned}
$$

we have $H_{6}+H_{8} \leq 0$ from Lemma 3.3. Moreover, we have $H_{2}+H_{4} \leq-\sum_{\ell \in A^{1}} \lambda_{t \ell}^{1} H_{1}$ since $z_{t-1}^{1}\left(i_{1}, i_{2}\right) \leq z_{t-1}\left(i_{1}-1, i_{2}\right)$. Similarly, we have $H_{7}+H_{9} \leq 0$ since $z_{t-1}^{2}\left(i_{1}-2, i_{2}\right) \geq$ $z_{t-1}^{2}\left(i_{1}-1, i_{2}\right)$. Furthermore, since $z_{t-1}^{2}\left(i_{1}, i_{2}\right) \leq z_{t-1}^{2}\left(i_{1}-1, i_{2}\right)$, we have

$$
\begin{aligned}
H_{3}+H_{5} & \leq \sum_{\ell \in A^{2}} \lambda_{t \ell}^{2}\left(z_{t-1}^{2}\left(i_{1}-1, i_{2}\right)-z_{t-1}^{2}\left(i_{1}, i_{2}\right)\right) \\
& =\sum_{\ell \in A^{2}} \lambda_{t \ell}^{2}\left(-H_{1}+F_{t-1}\left(i_{1}, i_{2}-1\right)-F_{t-1}\left(i_{1}-1, i_{2}-1\right)\right) \leq-\sum_{\ell \in A^{2}} \lambda_{t \ell}^{2} H_{1}
\end{aligned}
$$

Therefore, $z_{t}^{1}\left(i_{1}, i_{2}\right)-z_{t}^{1}\left(i_{1}-1, i_{2}\right) \leq\left(1-\sum_{\ell \in A^{1}} \lambda_{t \ell}^{1}-\sum_{\ell \in A^{2}} \lambda_{t \ell}^{2}\right) H_{1} \leq 0$.

Similarly, we can show that $z_{t}^{2}\left(i_{1}, i_{2}\right)-z_{t}^{2}\left(i_{1}, i_{2}-1\right) \leq 0$. Thus, we have completed the proof.

\section{Proof of Theorm 3.3}

Proof: First, by Lemma 3.4 we have

$$
\begin{aligned}
z_{t-1}^{1}\left(1, i_{2}\right) & =v_{t-1}\left(1, i_{2}\right)-v_{t-1}\left(0, i_{2}\right) \\
& \leq v_{t-1}\left(0, i_{2}+1\right)-v_{t-1}\left(0, i_{2}\right)=z_{t-1}^{2}\left(0, i_{2}+1\right) \leq z_{t-1}^{2}\left(0, i_{2}\right) .
\end{aligned}
$$

Furthermore, we have $z_{t-1}^{1}\left(i_{1}, i_{2}\right) \leq z_{t-1}^{1}\left(i_{1}-1, i_{2}\right)$ by Lemma 3.2 and $z_{t-1}^{2}\left(i_{1}, i_{2}\right) \leq z_{t-1}^{2}\left(i_{1}-\right.$ $1, i_{2}$ ) by Lemma 3.3 , thus by $(5)$ and Lemma 3.1 we obtain

$$
\begin{aligned}
z_{t}^{1}\left(i_{1}, i_{2}\right)-z_{t-1}^{1}\left(i_{1}, i_{2}\right) & \\
= & K_{t}^{1}\left(z_{t-1}^{1}\left(i_{1}, i_{2}\right)\right)-K_{t}^{1}\left(z_{t-1}^{1}\left(i_{1}-1, i_{2}\right)\right) I\left(i_{1} \geq 2\right)-K_{t}^{1}\left(z_{t-1}^{2}\left(0, i_{2}\right)\right) I\left(i_{1}=1\right) \\
& +K_{t}^{2}\left(z_{t-1}^{2}\left(i_{1}, i_{2}\right)\right) I\left(i_{2} \geq 1\right)-K_{t}^{2}\left(z_{t-1}^{2}\left(i_{1}-1, i_{2}\right)\right) I\left(i_{2} \geq 1\right) \geq 0 .
\end{aligned}
$$

Similarly, we can show that $z_{t}^{2}\left(i_{1}, i_{2}\right)-z_{t-1}^{2}\left(i_{1}, i_{2}\right) \geq 0$. Therefore, we have completed the proof. 


\section{References}

[1] J. Alstrup, S. Boas, O.B.G. Madsen and R.V.V. Vidal: Booking policy for flights with two types of passengers. European Journal of Operational Research, 27 (1986) 274-288.

[2] P. P. Belobaba: Survey Paper: Airline yield management an overview of seat inventory control. Transportation Science, 21 (1987) 63-73.

[3] P. P. Belobaba: Application of a probabilistic decision model to airline seat inventory control. Operations Research, 37 (1989) 183-197.

[4] S. L. Brumelle, J. I. McGill, T. H. Oum, K. Sawaki and M. W. Tretheway: Allocation of Airline Seats between Stochastically Dependent Demands. Transportation Science, $\mathbf{2 4}$ (1990) 183-192.

[5] S. L. Brumelle and J. I. McGill: Airline seat allocation with multiple nested fare classes. Operations Research, 41 (1993) 127-137.

[6] R. E. Curry: Optimal airline seat allocation with fare classes nested by origins and destination. Transportation Science, 24 (1990) 193-204.

[7] Y. Gerchak, M. Parlar and T. Yee: Optimal Rationing policies and production quantities for products with several demand classes. Canadian Journal of Administrative Science, 1 (1985) 161-176.

[8] C. J. Lautenbacher and S. Stidhain, Jr.: The underlying Markov decision process in the single-leg airline yield-management problem. Transportation Science, 33 (1999) 136-146.

[9] J. I. McGiLL and G. J. van Ryzin: Revenue management: Research overview and prospects. Transportation Science, 33 (1999) 233-256.

[10] T. L. Lee and M. Hersh: A model for dynamic airline seat inventory control with multiple seat bookings. Transportation Science, 27 (1993) 252-265.

[11] K. Littlewood: Forecasting and control of passenger bookings. Airline Group International Federation of Operational Research Societies Proceedings, 12 (1972) 95-117.

[12] L. W. Robinson: Optimal and approximate control policies for airline booking with sequential nonmonotonic fare classes. Operations Research, 43 (1995) 252-263.

[13] L. R. Weatherford, S. E. Bodily, and P. E. Pfeifer: Modeling the customer arrival process and comparing decision rules in perishable asset revenue management situations. Transportation Science, 27 (1993) 239-251.

[14] L. R. Weatherford and S. E. Bodily: A taxonomy and research overview of perishableasset revenue management: Yield management, overbooking, and pricing. Operations Research, 40 (1992) 831-844.

[15] R. D. Wollmer: An airline seat management model for a single leg route when lower fare classes book first. Operations Research, 40 (1992) 26-37.

[16] P. S. You: Dynamic pricing in airline seat management for flights with multiple flight legs. Transportation S'cience, 33 (1999) 192-206.

[17] P. S. You: Airline seat management with rejection-for-possible-upgrade decision. Transportation Research B, 35 (2001) 507-524.

Peng-Sheng You

Department of Industrial Management

National Taiwan University of Science and Technology

43, Keelung Rd., Sec. 4, Taipei, 106

Taipei, 106, TAIWAN

E-mail: yuu@im.ntust.edu.tw 